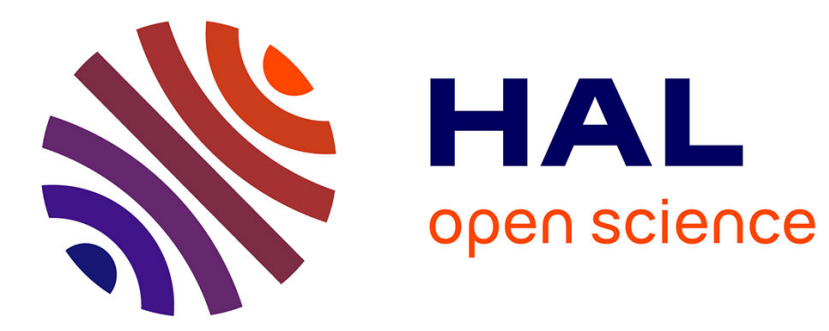

\title{
On the infinity-norm of the cubic form of complete hyperbolic affine hyperspheres
}

\author{
Roland Hildebrand
}

\section{To cite this version:}

Roland Hildebrand. On the infinity-norm of the cubic form of complete hyperbolic affine hyperspheres. Results in mathematics = Resultate der Mathematik, 2013, 64 (1-2), pp.113-119. 10.1007/s00025012-0301-7 . hal-00944369

\section{HAL Id: hal-00944369 https://hal.science/hal-00944369}

Submitted on 10 Feb 2014

HAL is a multi-disciplinary open access archive for the deposit and dissemination of scientific research documents, whether they are published or not. The documents may come from teaching and research institutions in France or abroad, or from public or private research centers.
L'archive ouverte pluridisciplinaire HAL, est destinée au dépôt et à la diffusion de documents scientifiques de niveau recherche, publiés ou non, émanant des établissements d'enseignement et de recherche français ou étrangers, des laboratoires publics ou privés. 


\title{
On the $\infty$-norm of the cubic form of complete hyperbolic affine hyperspheres
}

\author{
Roland Hildebrand *
}

November 14, 2011

\begin{abstract}
Let $M_{n} \subset \mathbb{R}^{n+1}$ be a complete hyperbolic affine hypersphere with mean curvature $H, H<0$, and let $C$ be its cubic form. We derive a differential inequality and an upper bound on the scalar function $\|C\|_{\infty}$ defined by the fiber-wise maximum of the value of $C$ on the unit sphere bundle of $M$. The bound is attained for the affine hyperspheres which are asymptotic to a simplicial cone.
\end{abstract}

\section{Introduction}

The subject of this paper are complete hyperbolic affine hyperspheres. The Calabi conjecture [2] states that every hyperbolic affine hypersphere $M$ which is complete (in the affine metric or in a metric induced by some Euclidean metric of the ambient affine space) is asymptotic to a regular (with nonempty interior and containing no lines) convex cone in the ambient space, with vertex in the center of $M$, and conversely, for every regular convex cone $K$ in the ambient space and every negative real number $H$ there exists a unique hyperbolic affine hypersphere with center in the vertex of $K$ and with mean curvature $H$ which is asymptotic to $K$. The conjecture has been proven by the efforts of many authors, a synthesis of the proof is given in [5, Section 2].

In [2, Lemma 5.2, p.31] Calabi obtained a differential inequality on the maximal eigenvalue of the Ricci tensor on complete hyperbolic affine hyperspheres and proved that the Ricci curvature has to be non-positive [2, Theorem 5.1, p.31]. He also showed that this bound is sharp, by presenting an example of a complete hyperbolic affine hypersphere with flat affine metric, namely the one which is asymptotic to a simplicial cone [2, p.37]. Similar differential inequalities and bounds can be obtained for the Pick invariant [9, eq. (2.5)], [5, Cor. 2.6.5, p.128].

In this contribution we use the techniques of [2] to obtain a differential inequality (Theorem 3.1) and a bound (Corollary 4.1) on the function

$$
\Upsilon(x)=\max _{\xi \in T_{x} M,\|\xi\|=1}(C(x))(\xi, \xi, \xi)
$$

on a complete hyperbolic affine hypersphere $M$, with $C$ being the cubic form. This function can be considered as the (point-wise) $\infty$-norm of the cubic form. The main motivation for studying this function lies in conic optimization, to which a link can be made as follows. An $n$-dimensional complete hyperbolic affine hypersphere $M$ with center in the origin of $\mathbb{R}^{n+1}$ is asymptotic to some regular convex cone $K \subset \mathbb{R}^{n+1}$. On the interior of $K$ define a logarithmically homogeneous convex function $F$ by the relation $F[\alpha M]=\{-\log \alpha\}$ for all $\alpha>0$ (cf. [6]). Then a bound on (1) translates into a bound on the self-concordance parameter [7, Sect. 2.3.3] of an appropriate multiple of $F$. We will elaborate on this relation in a subsequent publication (cf. also [4, Theorem 4.8]). Our bound on the cubic form is sharp, as we will demonstrate on the example of the affine hypersphere asymptotic to a simplicial cone (Proposition 5.1).

${ }^{*}$ LJK, Université Grenoble 1 / CNRS, 51 rue des Mathématiques, BP53, 38041 Grenoble cedex, France (roland.hildebrand@imag.fr). 


\section{Notations and preliminaries}

Let us recall the definition of the cubic form and the expression for its Laplacian on affine hyperspheres. Let $M$ be an $n$-dimensional complete hyperbolic affine hypersphere with mean curvature $H<0, g$ the affine metric, $\nabla$ the Levi-Civita connection of the affine metric, and $\bar{\nabla}$ the affine connection induced by the ambient real affine space. The cubic form is defined as the covariant derivative $C=\bar{\nabla} g[8$, eq. (2.6), p.34; Theorem 3.3, p.42]. The difference tensor ${ }^{1}$ is defined as the difference $\bar{\nabla}-\nabla$ and equals $A=-\frac{1}{2} C[8$, Prop.4.1, p.50]. The difference tensor and hence the cubic form are symmetric in all three indices [2, pp.23-24]. The Ricci curvature of the affine metric and the Laplacian of $C$ with respect to the affine metric are given by $[9, \mathrm{p} .3]$

$$
\begin{aligned}
R_{i j} & =(n-1) H g_{i j}+\frac{1}{4} C_{i}^{r s} C_{j r s} \\
\Delta C_{i j k} & =(n+1) H C_{i j k}+\frac{1}{4} C^{a b c}\left(C_{a b i} C_{c j k}+C_{a b j} C_{c k i}+C_{a b k} C_{c i j}\right)-\frac{1}{2} C_{i b}^{a} C_{j c}^{b} C_{k a}^{c} .
\end{aligned}
$$

\section{Differential inequality}

Our purpose is to derive a differential inequality on the function $\Upsilon$ defined by (1) on $M$. Let $p \in M$ be an arbitrary point and let $\xi \in T_{p} M$ be a maximizer of the cubic form on the unit sphere in $T_{p} M$, $\Upsilon(p)=(C(p))(\xi, \xi, \xi),\|\xi\|=1$.

Transport $\xi$ from $p$ to a neighbourhood $U$ of $p$ along the geodesics through $p$ by means of the Levi-Civita connection to obtain a smooth unit length vector field on $U$. Then $\xi$ satisfies

$$
\nabla \xi=0, \quad \Delta \xi=0
$$

at $p\left[2\right.$, p.32], and $\bar{\Upsilon}=C_{i j k} \xi^{i} \xi^{j} \xi^{k} \leq \Upsilon$ on $U$, with equality attained at $p$. Define the symmetric second-order tensor $B=\bar{\nabla}_{\xi} g$ on $U$, in index form $B_{i j}=C_{i j k} \xi^{k}$. Note that $B$ is traceless by the apolarity condition $C_{i j}^{i}=0\left[8\right.$, Theorem 4.3, p.51], and that $B_{i j} \xi^{i} \xi^{j}=\bar{\Upsilon}$. Moreover, by [5, Lemma 2.2.3.19, p.106] we have at $p$ that $B_{i j} \xi^{i} \eta^{j}=0$ and $B_{i j} \eta^{i} \eta^{j} \leq \frac{1}{2} B_{i j} \xi^{i} \xi^{j} \leq \bar{\Upsilon}$ for every unit length vector $\eta$ which is orthogonal to $\xi$. It follows that $\xi$ is also a maximizer of $B(p)$ on the unit sphere in $T_{p} M$. In particular, at $p$ we have

$$
B_{i j} \xi^{j}=\bar{\Upsilon} g_{i j} \xi^{j}
$$

as the first order optimality condition, and $\bar{\Upsilon}$ is the maximal eigenvalue of the matrix of $B(p)$ in any orthonormal basis of $T_{p} M$.

Let us now estimate the Laplacian of $\bar{\Upsilon}$. At $p$ we have by virtue of $(3),(4)$, and (5)

$$
\begin{aligned}
\Delta \bar{\Upsilon} & =(\Delta C)_{i j k} \xi^{i} \xi^{j} \xi^{k}=(n+1) H \bar{\Upsilon}+\frac{3}{4} C^{a b c} C_{a b i} C_{c j k} \xi^{i} \xi^{j} \xi^{k}-\frac{1}{2} C_{i b}^{a} C_{j c}^{b} C_{k a}^{c} \xi^{i} \xi^{j} \xi^{k} \\
& =(n+1) H \bar{\Upsilon}+\frac{3}{4} C^{a b c} B_{a b} B_{c j} \xi^{j}-\frac{1}{2} B_{b}^{a} B_{c}^{b} B_{a}^{c}=(n+1) H \bar{\Upsilon}+\frac{3}{4} \bar{\Upsilon} B^{a b} B_{a b}-\frac{1}{2} B_{b}^{a} B_{c}^{b} B_{a}^{c} \\
& \geq(n+1) H \bar{\Upsilon}+\frac{n(n+1)}{4(n-1)^{2}} \bar{\Upsilon}^{3} .
\end{aligned}
$$

Here the inequality follows from Corollary A.2 in the Appendix. We obtain the following result.

Theorem 3.1. Let $M$ be an $n$-dimensional complete hyperbolic affine hypersphere with mean curvature $H<0$, and let $C$ be its cubic form. Then the function $\Upsilon(x)=\max _{\xi \in T_{x} M,\|\xi\|=1}(C(x))(\xi, \xi, \xi)$ satisfies the differential inequality $\Delta \Upsilon \geq(n+1) H \Upsilon+\frac{n(n+1)}{4(n-1)^{2}} \Upsilon^{3}$ weakly in the sense of $[1$, Def. 1, p.46].

\footnotetext{
${ }^{1}$ Sometimes, such as in [2], the difference tensor is called cubic form, which can lead to serious confusions and apparently resulted in a missing factor of 2 in [3, Cor. 2, p.857] for the bound on the Pick invariant.
} 


\section{Bound}

From Theorem 3.1 we might obtain a bound on the function (1) on a complete hyperbolic affine hypersphere. Namely, from [3, Cor. 1, p.857 $]^{2}$ it follows that $\Upsilon \leq \sqrt{-\frac{4 H(n-1)^{2}}{n}}=\frac{2(n-1) \sqrt{-H}}{\sqrt{n}}$. As in the case of the Pick invariant [5, Cor. 2.6.5, p.128], however, there exists a purely algebraic proof.

As in the preceding section, let $p \in M$ be an arbitrary point, let $\xi \in T_{p} M$ be a maximizer of the cubic form on the unit sphere in $T_{p} M$, and define the traceless symmetric second-order tensor $B=\bar{\nabla}_{\xi} C$ on $T_{p} M$. Let $\left\{\xi, \eta_{1}, \ldots, \eta_{n-1}\right\}$ be an orthonormal basis of $T_{p} M$, then we have

$$
\begin{aligned}
\Upsilon^{2}(p) & =\left(B_{i j} \xi^{i} \xi^{j}\right)^{2}=\left(-\sum_{k=1}^{n-1} B_{i j} \eta_{k}^{i} \eta_{k}^{j}\right)^{2} \leq(n-1) \sum_{k=1}^{n-1}\left(B_{i j} \eta_{k}^{i} \eta_{k}^{j}\right)^{2} \leq(n-1) \sum_{k=1}^{n-1} B_{i l} B_{j}^{l} \eta_{k}^{i} \eta_{k}^{j} \\
& =(n-1)\left(B_{i l} B^{l i}-B_{i l} B_{j}^{l} \xi^{i} \xi^{j}\right)=(n-1)\left(C_{i l j} C_{k}^{l i} \xi^{j} \xi^{k}-\Upsilon^{2}(p)\right) \\
& =4(n-1)\left(R_{j k}-(n-1) H g_{j k}\right) \xi^{j} \xi^{k}-(n-1) \Upsilon^{2}(p) \leq-4(n-1)^{2} H-(n-1) \Upsilon^{2}(p) .
\end{aligned}
$$

Here we used (5) and (2) in the last two equalities, respectively, and the non-positivity of the Ricci curvature [2, Theorem 5.1, p.31] in the last inequality. We obtain the following result.

Corollary 4.1. Let $M$ be an n-dimensional complete hyperbolic affine hypersphere with mean curvature $H<0$, and let $C$ be its cubic form. Then the function $\Upsilon(x)=\max _{\xi \in T_{x} M,\|\xi\|=1}(C(x))(\xi, \xi, \xi)$ satisfies the inequality $\Upsilon \leq \frac{2(n-1) \sqrt{-H}}{\sqrt{n}}$.

\section{Affine hyperspheres asymptotic to a simplicial cone}

In this section we show that the inequalities in Theorem 3.1 and Corollary 4.1 are saturated for the affine hyperspheres which are asymptotic to a simplicial cone.

Denote by $I_{k}$ the $k \times k$ identity matrix, by $\mathbf{1}_{k} \in \mathbb{R}^{k}$ the all-ones vector, and by $e_{k} \in \mathbb{R}^{n}$ the $k$-th canonical basis vector. Let $K=\mathbb{R}_{+}^{n+1}$ be the nonnegative orthant, $H<0$ a negative real number, and let $M \subset K$ be the hyperbolic affine hypersphere with mean curvature $H$ which is asymptotic to $K$. It is well-known that $M$ is given by the equation $x_{0} \cdots x_{n}=c$ for some $c>0$. On the interior of $K$ we introduce the coordinates $y_{0}, \ldots, y_{n}$ by

$$
\left(\begin{array}{c}
y_{0} \\
\vdots \\
y_{n}
\end{array}\right)=\gamma U \log x=\gamma\left(\begin{array}{cc}
\frac{1}{\sqrt{n+1}} & \frac{1}{\sqrt{n+1}} \mathbf{1}_{n}^{T} \\
-\frac{1}{\sqrt{n+1}} \mathbf{1}_{n} & I_{n}+\frac{1}{n}\left(\frac{1}{\sqrt{n+1}}-1\right) \mathbf{1}_{n} \mathbf{1}_{n}^{T}
\end{array}\right)\left(\begin{array}{c}
\log x_{0} \\
\vdots \\
\log x_{n}
\end{array}\right)
$$

where $\gamma>0$ is a constant to be determined later. Then in the $y$-coordinates the surface $M$ is given by the equation $y_{0}=\frac{\gamma \log c}{\sqrt{n+1}}$. The remaining coordinates $y_{1}, \ldots, y_{n} \in \mathbb{R}$ define a global coordinate chart on $M$.

It is easily seen that the subgroup of unimodular diagonal automorphisms of $K$, which leaves $M$ invariant, acts by translations on $M$. The affine metric $g$ and the cubic form $C$ of $M$ have thus constant coefficients. The symmetric group $S_{n+1}$, which acts by permutations of the coordinates $x_{i}$ on $K$, has a unique fixed point $y^{0} \in M$ given by $y_{1}=\cdots=y_{n}=0$. Therefore $S_{n+1}$ acts on the tangent space $T_{y^{0}} M$, and both $g$ and $C$ have to be invariant under this action. It is not hard to check that the subgroup $S_{n} \subset S_{n+1}$ permuting the coordinates $x_{1}, \ldots, x_{n}$ on $K$ acts just by a corresponding permutation of the

\footnotetext{
${ }^{2}$ Obviously, in the formulation of this Corollary it should read $d_{1}>0, d_{2}<0$, and $u \leq \sqrt{-d_{2} / d_{1}}$, otherwise the assertion of the Corollary can be disproved simply by choosing $u$ to be an appropriate constant. The proof of the Corollary contains several flaws, e.g., it assumes that a lower bound on the Ricci curvature implies an upper bound on the quantity $r \Delta r$, where $r$ is the distance function from some point $p$ [3, p.856]. Consider the unit ball in $\mathbb{R}^{n}$ equipped with the hyperbolic geometry given by the metric $g(x)=\frac{\left(1-R^{2}\right) I+x x^{T}}{\left(1-R^{2}\right)^{2}}$ in matrix form, where $R=\sqrt{x^{T} x}$ is the Euclidean distance from the centre and $I$ the identity matrix. The geodesic distance function from the centre is then given by $r=\frac{1}{2} \log \frac{1+R}{1-R}$, and $\Delta r=\frac{n-1}{R}$. Hence $r \Delta r$ tends to $+\infty$ as $R \rightarrow 1$. It is, however, straightforward to write down a correct proof of the Corollary, e.g., by setting $f=\left(a^{2}-r^{2}\right) u\left[3\right.$, p.856]. With the choice $f=\left(1+\cos \frac{\pi r^{2}}{a^{2}}\right) u$ one can handle also differential inequalities of the form $\Delta \psi \geq d_{1} u^{2}+d_{2} u$.
} 
$y$-coordinates. Therefore the coefficients $g_{i j}, C_{i j k}$ depend only on the number of distinct indices in the sets $\{i, j\}$ and $\{i, j, k\}$, respectively. Denote the latter number by $\theta_{i j k}$. The permutation $\sigma_{i} \in S_{n+1}$ exchanging the coordinates $x_{0}, x_{i}$ acts on $T_{y^{0}} M$ by the orthogonal transformation

$$
U_{i}=I-\left(\frac{1-\sqrt{n+1}}{n} \mathbf{1}_{n}-e_{i}\right)\left(\frac{1-\sqrt{n+1}}{n} \mathbf{1}_{n}-e_{i}\right)^{T}
$$

It is not difficult to check that the invariance of $g$ and $C$ with respect to the transformations given by $U_{i}$ determines these tensors up to a constant multiple. Namely, the matrix of the metric $g$ has to be proportional to the identity matrix, and the cubic form $C$ is given by

$$
C_{i j k}=\left\{\begin{array}{rlrl}
-\alpha(n-1)(2+(n-2) \sqrt{n+1}), & & \theta_{i j k}=1, \\
\alpha(2+(n-2) \sqrt{n+1}), & \theta_{i j k}=2, \\
\alpha(n+2-2 \sqrt{n+1}), & \theta_{i j k}=3,
\end{array}\right.
$$

where $\alpha$ is a yet to be determined proportionality constant.

Let us choose $\gamma$ such that the matrix of $g$ is given by $I_{n}$. Contracting (2) with the metric and taking into account that $M$ is flat, we obtain $C^{i r s} C_{i r s}=-4 n(n-1) H$. Inserting the values for $C_{i r s}$ from (6), we get $\alpha^{2} n^{5}(n-1)=-4 n(n-1) H$, yielding $\alpha= \pm \frac{2 \sqrt{-H}}{n^{2}}$ (it can be checked that the sign is positive). Hence on the unit length vector $\frac{1}{\sqrt{n}} \mathbf{1}_{n}$ the cubic form has the value $\frac{\alpha n^{3}(n-1)}{n^{3 / 2}}= \pm \frac{2(n-1) \sqrt{-H}}{\sqrt{n}}$, finally giving $\Upsilon \geq \frac{2(n-1) \sqrt{-H}}{\sqrt{n}}$. From Corollary 4.1 we can then conclude that $\Upsilon \equiv \frac{2(n-1) \sqrt{-H}}{\sqrt{n}}$ on $M$. By the affine equivalence of an arbitrary simplicial cone to the nonnegative orthant we obtain the following result.

Proposition 5.1. Let $M \subset \mathbb{R}^{n+1}$ be a complete hyperbolic affine hypersphere which is asymptotic to a simplicial cone. Then both the bound in Theorem 3.1 and in Corollary 4.1 are saturated.

In particular, this will allow us to show that the optimal self-concordance parameter of an arbitrary regular convex cone is not worse than the parameter of the standard barrier for the nonnegative orthant of the same dimension.

\section{References}

[1] Eugenio Calabi. An extension of E. Hopf's maximum principle with an application to Riemannian geometry. Duke Math. J., 25:45-56, 1958.

[2] Eugenio Calabi. Complete affine hyperspheres I. In Symposia Mathematica Vol. 10, pages 19-38. Istituto Nazionale di Alta Matematica, Acad. Press, 1972.

[3] S.Y. Cheng and S.T. Yau. Complete affine hypersurfaces, part I. The completeness of affine metrics. Comm. Pure Appl. Math., 39:839-866, 1986.

[4] Roland Hildebrand. Barriers on projective convex sets, 2011. accepted.

[5] An-Min Li, Udo Simon, and Guosong Zhao. Global affine differential geometry of hypersurfaces, volume 11 of De Gruyter expositions in mathematics. Walter de Gruyter, 1993.

[6] John C. Loftin. Affine spheres and Kähler-Einstein metrics. Math. Res. Lett., 9(4):425-432, 2002.

[7] Yurii Nesterov and Arkadii Nemirovskii. Interior-point polynomial algorithms in convex programming, volume 13 of SIAM Stud. Appl. Math. SIAM, Philadelphia, 1994.

[8] Katsumi Nomizu and Takeshi Sasaki. Affine differential geometry: geometry of affine immersions, volume 111 of Cambridge Tracts in Mathematics. Cambridge University Press, Cambridge, 1994.

[9] Rolf Schneider. Zur affinen Differentialgeometrie im Grossen II. über eine Abschätzung der Pickschen Invariante auf Affinsphären. Math. Z., 102:1-8, 1967. 


\section{A Matrix lemma}

In this section we provide an auxiliary inequality.

Lemma A.1. Let $n \geq 2$ and $\lambda_{1}, \ldots, \lambda_{n-1} \leq 1$ be such that $\sum_{i=1}^{n-1} \lambda_{i}=-1$. Then $\frac{3}{4} \sum_{i=1}^{n-1} \lambda_{i}^{2}-$ $\frac{1}{2} \sum_{i=1}^{n-1} \lambda_{i}^{3} \geq \frac{3 n-1}{4(n-1)^{2}}$.

Proof. Define $c_{i}=(n-1) \lambda_{i}+1$. Then $\sum_{i=1}^{n-1} c_{i}=0$ and $c_{i} \leq n$ for all $i$. It follows that $c_{i}^{3} \leq n c_{i}^{2}$ for all $i$. We then have

$$
\begin{aligned}
& \frac{3}{4} \sum_{i=1}^{n-1} \lambda_{i}^{2}-\frac{1}{2} \sum_{i=1}^{n-1} \lambda^{3}-\frac{3 n-1}{4(n-1)^{2}}=\frac{3}{4(n-1)^{2}} \sum_{i=1}^{n-1}\left(c_{i}-1\right)^{2}-\frac{1}{2(n-1)^{3}} \sum_{i=1}^{n-1}\left(c_{i}-1\right)^{3}-\frac{3 n-1}{4(n-1)^{2}} \\
& =\left(\frac{3}{4(n-1)}+\frac{1}{2(n-1)^{2}}-\frac{3 n-1}{4(n-1)^{2}}\right)-\left(\frac{3}{2(n-1)^{2}}+\frac{3}{2(n-1)^{3}}\right) \sum_{i=1}^{n-1} c_{i} \\
& \quad+\left(\frac{3}{4(n-1)^{2}}+\frac{3}{2(n-1)^{3}}\right) \sum_{i=1}^{n-1} c_{i}^{2}-\frac{1}{2(n-1)^{3}} \sum_{i=1}^{n-1} c_{i}^{3} \\
& \geq\left(\frac{3(n+1)}{4(n-1)^{3}}-\frac{n}{2(n-1)^{3}}\right) \sum_{i=1}^{n-1} c_{i}^{2}=\frac{n+3}{4(n-1)^{3}} \sum_{i=1}^{n-1} c_{i}^{2} \geq 0 .
\end{aligned}
$$

This completes the proof.

Corollary A.2. Let $B$ be a real symmetric $n \times n$ matrix with vanishing trace. Then $\frac{3}{4} \lambda_{\max }(B) \operatorname{tr} B^{2}-$ $\frac{1}{2} \operatorname{tr} B^{3} \geq \frac{n(n+1)}{4(n-1)^{2}} \lambda_{\max }^{3}(B)$.

Proof. If $\lambda_{\max }(B)=0$, then $B=0$ and the assertion of the corollary is evident.

Suppose now that $\lambda_{\max }(B)>0$. Let $\lambda_{1} \leq \lambda_{2} \leq \cdots \leq \lambda_{n-1} \leq \lambda_{n}=1$ be the eigenvalues of the matrix $\tilde{B}=\frac{B}{\lambda_{\max }(B)}$ in increasing order. Then $\sum_{i=1}^{n-1} \lambda_{i}=-1$ and $\lambda_{i} \leq 1$ for all $i$. We then have

$$
\begin{aligned}
& \frac{3}{4} \lambda_{\max }(B) \operatorname{tr} B^{2}-\frac{1}{2} \operatorname{tr} B^{3}-\frac{n(n+1)}{4(n-1)^{2}} \lambda_{\max }^{3}(B)=\lambda_{\max }^{3}(B)\left(\frac{3}{4} \operatorname{tr} \tilde{B}^{2}-\frac{1}{2} \operatorname{tr} \tilde{B}^{3}-\frac{n(n+1)}{4(n-1)^{2}}\right) \\
& \quad=\lambda_{\max }^{3}(B)\left[\frac{3}{4}\left(1+\sum_{i=1}^{n-1} \lambda_{i}^{2}\right)-\frac{1}{2}\left(1+\sum_{i=1}^{n-1} \lambda_{i}^{3}\right)-\frac{n(n+1)}{4(n-1)^{2}}\right] \geq 0
\end{aligned}
$$

where the inequality comes from the preceding lemma. 